\title{
Influência da Hipoterapia no Equilíbrio Estático em Um Indivíduo com Doença de Charcot-Marie-Tooth
}

\author{
Hippotherapy's Influence In Static Balance In a Subject with Charcot-Marie-Tooth Disease \\ Cristiane Helita Zorél Meneghetti ${ }^{1}$, Miriam Tonon de Meira ${ }^{2}$, Sofia \\ Poletti ${ }^{3}$, Ana Carolina Teixeira Batistela ${ }^{4}$ Luiz Carlos Ferracini Junior ${ }^{5}$
}

\begin{abstract}
RESUMO
Objetivo. Verificar a influência da hipoterapia no equilíbrio estático em um indivíduo com doença de Charcot-Marie-Tooth (CMT). Método. Foi realizado um estudo de caso, com uma participante com doença de Charcot-Marie-Tooth, gênero feminino, 12 anos de idade. A participante foi filmada na vista anterior (plano frontal) e perfil (plano sagital). O instrumento utilizado foi a Biofotogrametria Computadorizada que serviu como referência angular para verificar as oscilaçóes do corpo em equilíbrio estático. A intervenção da hipoterapia foi realizada durante 16 sessôes, duas vezes por semana, num período de 2 meses, empregando o programa de hipoterapia e atividades eqüestres. Resultados. Os graus de oscilações avaliados após a intervenção da hipoterapia apresentaram diminuição tanto no plano frontal como no plano sagital. Conclusáo. O presente estudo mostrou que com a intervençáo da hipoterapia a participante com doença de Charcot Marie Tooth apresentou melhora em seu equilíbrio estático.
\end{abstract}

Unitermos. Equilíbrio Postural, Hipoterapia, Doença de CharcotMarie-Tooth, Fotogrametria.

Citaçáo. Meneghetti CHZ, Meira MT, Poletti S, Batistela ACT, Ferracini Junior LC. Influência da Hipoterapia no Equilíbrio Estático em Um Indivíduo com Doença de Charcot-Marie-Tooth.
Trabalho Realizado no Centro Universitário Hermínio Ometto - UNIARARAS, Araras-SP, Brasil.

1.Fisioterapeuta Mestre em Distúrbios do Desenvolvimento - MACKENZIE, São Paulo-SP, Brasil.

2.Fisioterapeuta, Especialista em Ortopedia - UNICAMP, Campinas-SP, Brasil. 3.Fisioterapeuta, Docente da UNIARARAS, Araras-SP, Brasil.

4.Fisioterapeuta, Mestre em Fisioterapia - UNIMEP, Piracicaba-SP, Brasil.

5.Fisioterapeuta, Mestrando em Ciências Biomédicas - UNIARARAS, ArarasSP, Brasil.

\begin{abstract}
Objective. Verify hippotherapy's influence in static balance in a subject with Charcot-Marie-Tooth (CMT). Method. It was done a study of case, with one subject with Charcot-Marie-Tooth, female gender, 12 years old. The subject was filmed in anterior view (frontal view) and profile (saggital view). The instrument used was Computerized Biophotogrammetry which served as angular reference to verify body's oscillations in the static balance. The hippotherapy was done during 16 sessions, twice a week, for 2 months, using the hippotherapy program and equestrian activities. Results. The oscillations's degrees evaluated after the hippotherapy intervention showed decrease both in frontal and saggital views. Conclusion. This study showed that with the hippotherapy intervention this subject with CharcotMarie-Tooth showed improvement in her static balance.
\end{abstract}

Keywords. Postural Balance, Hippotherapy, Charcot-Marie-Tooth Disease, Photogrammetry.

Citation. Meneghetti CHZ, Meira MT, Poletti S, Batistela ACT, Ferracini Junior LC. Hippotherapy's Influence In Static Balance In a Subject with Charcot-Marie-Tooth Disease. 


\section{INTRODUÇÃO}

A doença de Charcot-Marie-Tooth (CMT) é a mais frequente forma de doenças neuromusculares hereditária do sistema nervoso periférico, podendo ser herdada geneticamente ou ocorrer por uma mutação genéti$\mathrm{Ca}^{1,2}$. É definida como uma neuropatia sensorial e motora hereditária, transmitida com mais frequência como traço autossômico dominante e da autossômica recessiva, mas também pode estar ligada ao cromossomo X, dominante ou recessiva ${ }^{3,4}$.

A classificação da doença de Charcot-Marie-Tooth se baseia na alteração de bainha de mielina tipo desmielinizantes (tipo I) e axonal (tipo II), principalmente nos distúrbios cromossômicos, e sua incidência é de 1:2.500 podendo afetar crianças e adultos ${ }^{2-5}$. A CMT provoca uma degeneraçáo lenta e progressiva dos nervos periféricos, levando a amiotrofia distal nos membros, fraqueza muscular, perda da sensibilidade nas extremidades do corpo e perda do mecanismo de controle postural5-7.

O controle postural possui dois objetivos comportamentais: a orientação e o equilíbrio. A orientação postural está relacionada ao posicionamento e ao alinhamento dos segmentos corporais um em relaçáo aos outros e em relação ao ambiente. $\mathrm{O}$ equilíbrio postural é o estado em que todas as forças que atuam sobre o corpo estão balanceadas para manter o corpo na posição e orientação desejada ${ }^{8}$. A estabilidade postural ou equilíbrio é definido como a capacidade de manter o centro da massa projetado dentro dos limites da base de apoio?.

Cavalgar é um processo de controle postural, que além de proporcionar a sensação de independência gera um aumento da autoconfiança. Na equoterapia há a participação do corpo inteiro do praticante, contribuindo em seu desenvolvimento. Durante a prática da hipoterapia a base de apoio do praticante é modificada, inibindo alguns padróes patológicos e com o cavalo ao passo recebe inúmeros estímulos que chegam ao Sistema Nervoso Central $^{10-12}$.

Os benefícios mais importantes recebidos pelo praticante de hipoterapia com o cavalo ao passo são: regularização tônica, coordenação motora, noção espacial, ritmo, flexibilidade, fortalecimento e estimulação proprioceptiva e vestibular ${ }^{10-14}$. Entre os instrumentos de avaliação do equilíbrio encontra-se a Biofotogrametria
Computadorizada, que se fundamenta na aplicação do princípio fotogramétrico às imagens fotográficas, obtidas de movimentos corporais. A essas imagens podem ser aplicadas bases de fotointerpretação, gerando uma nova ferramenta de estudo da cinemática ${ }^{15}$.

Desta forma, o objetivo do estudo foi analisar a influência da intervenção da Hipoterapia no equilíbrio estático de um indivíduo com doença de Charcot Marie Tooth.

\section{MÉTODO}

\section{Amostra}

Tratou-se de um modelo de estudo de caso, que obteve aprovação do Comitê de Ética e Mérito em Pesquisa sob o parecer $n^{\circ}$ 812/2008 do Centro Universitário Hermínio Ometto - Uniararas.

Participou do estudo um indivíduo com diagnóstico médico de doença de Charcot-Marie-Tooth (CMT), do gênero feminino, 12 anos de idade, apresentando um quadro motor de Diparesia com déficit de equilíbrio estático e dinâmico e dificuldades na marcha. Antes de iniciar a avaliação, o responsável assinou o Termo de Consentimento Livre e Esclarecido e consentiu na publicação da imagem.

\section{Procedimento}

A avaliação do equilíbrio estático ocorreu no laboratório de Biofotogrametria Computadorizada na Clínica Escola de Fisioterapia - Uniararas.

Foi colocado na participante um marcador adesivo na regiáo da glabela para avaliaçáo na vista anterior e sobre o eurio para avaliação em perfil. Durante a filmagem a participante foi orientada a manter uma postura relaxada, com os braços posicionados ao lado do corpo o mais estável possível, e os pés paralelos sobre uma superfície plana, previamente marcada para os apoios plantares, tendo ao fundo um fio de prumo, que serviu de referência para a análise do ângulo.

A participante foi filmada na vista anterior (plano frontal) e na vista de perfil direito (plano sagital).

$\mathrm{Na}$ filmagem na condição de olhos abertos foi pedido a participante que fixasse para um alvo formado por um papel amarelo em formato circular, posicionado na parede frontal da sala na altura dos olhos. A participante 
foi posicionada de modo que os pontos antropométricos previamente marcados com ponto adesivo de $19 \mathrm{~mm}$ coincidissem com o fio de prumo, na vista anterior (glabela) como no perfil direito (eurio). Para a formaçáo do ângulo, foi traçada uma reta ao vértice, perpendicular ao fio de prumo para determinação do ponto de interseção (Figura 1).

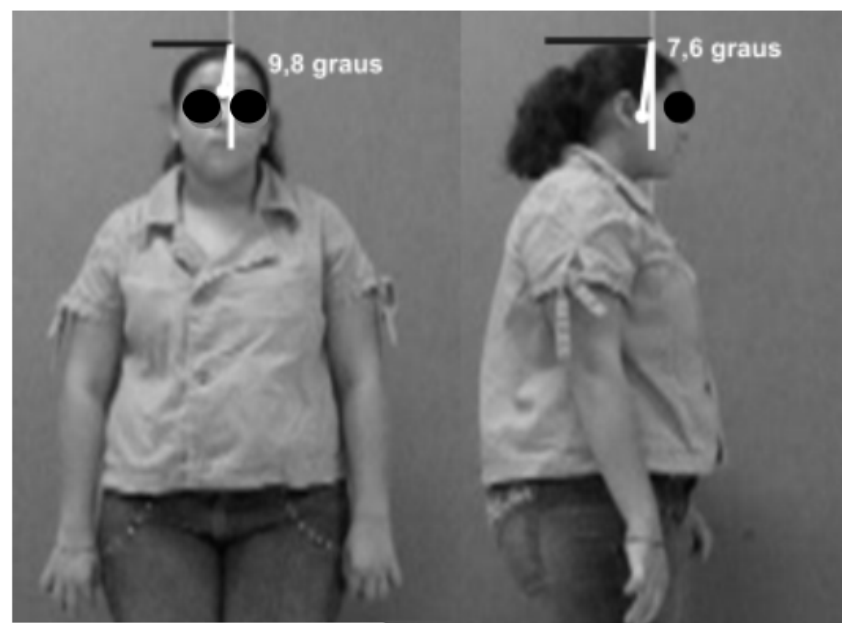

Figura 1. Quantificação angular em graus da oscilação na vista anterior (plano frontal) e na vista perfil (plano sagital), utilizando o software Corel Draw.

A câmera filmadora foi posicionada sobre um tripé com prumo de superfície e em nível, a uma distância de 2,37 metros e a uma altura de 93 centímetros do solo. O tempo de exposição nas filmagens foi de 30 segundos para cada postura.

A fim de se obter o momento de maior oscilação ântero-posterior (plano sagital) e latero-lateral (plano frontal), as imagens foram analisadas quadro a quadro pelo programa Windows Movie Maker, e no momento de maior oscilação de cada plano a imagem foi selecionada e analisada pelo software Corel Draw X3, através da Biofotogrametria Computadorizada, determinando-se os valores angulares em graus. As avaliações foram realizadas pelo mesmo avaliador sendo este treinado.

Após a avaliação iniciou-se a intervenção baseado na prática da Hipoterapia. As sessóes foram realizadas na Associação de Equoterapia de Araras (A.E.A), na cidade de Araras, São Paulo, utilizando-se um cavalo mestiço, lusitano, da raça quarto de milha, 8 anos de idade, $310 \mathrm{~kg}$, 1,52 m de altura, treinado para prática da Hipoterapia.
Ao total foram realizadas 16 sessões duas vezes por semana, durante 50 minutos, num período de dois meses. Cada sessão foi composta por alimentação do animal, escovação da pelagem, preparação para a montaria e montaria. Destinaram-se as duas primeiras sessóes para a adaptação do praticante ao contato com o animal. A montaria foi realizada sobre a manta com alça e estribo. A pista onde o trabalho de equoterapia foi realizado tinha a largura de 20 metros e 40 metros de comprimento. A intervenção foi através do programa de hipoterapia. Cada sessão de hipoterapia consistiu em variaçôes do andar do cavalo (passo e trote), de piso (areia e gramado), de terreno (plano, acidentado e inclinado), mudanças de direção e de combinações de movimentos utilizando formatos de figuras geométricas como: diagonais, círculos e retas.

Após a intervenção da hipoterapia a participante realizou uma nova avaliação do equilíbrio estático.

Nesse estudo foi proposta como forma de análise para quantificar os graus de oscilação ântero-posterior, a soma dos desvios da oscilação tanto anterior quanto posterior e na vista latero-lateral a soma das oscilaçóes para direita e para esquerda.

\section{RESULTADOS}

Verificou-se que os graus de oscilaçôes avaliados depois da intervenção da hipoterapia diminuíram tanto nos planos frontal como no sagital (Gráfico 1).

No plano frontal constatou-se uma reduçáo de 45,5 graus para 14,9 graus de oscilações, representando uma melhora da oscilação no plano frontal de 67,25\%. Da mesma forma, no plano sagital verificou-se uma redução de 33,2 graus para 14,4 graus de oscilação, proporcionando uma melhora no equilíbrio postural da participante analisada de 56,63\% na oscilação no plano sagital.

\section{DISCUSSÃO}

Pesquisas referentes à área do equilíbrio são importantes na busca do entendimento dessa complexa tarefa, uma vez que é fundamental para desenvolvimento de outras capacidades e para o desempenho funcional do ser humano.

De maneira geral, o resultado deste estudo mostrou que, após a intervenção da prática da Hipoterapia, a participante com doença de CMT apresentou diminui- 


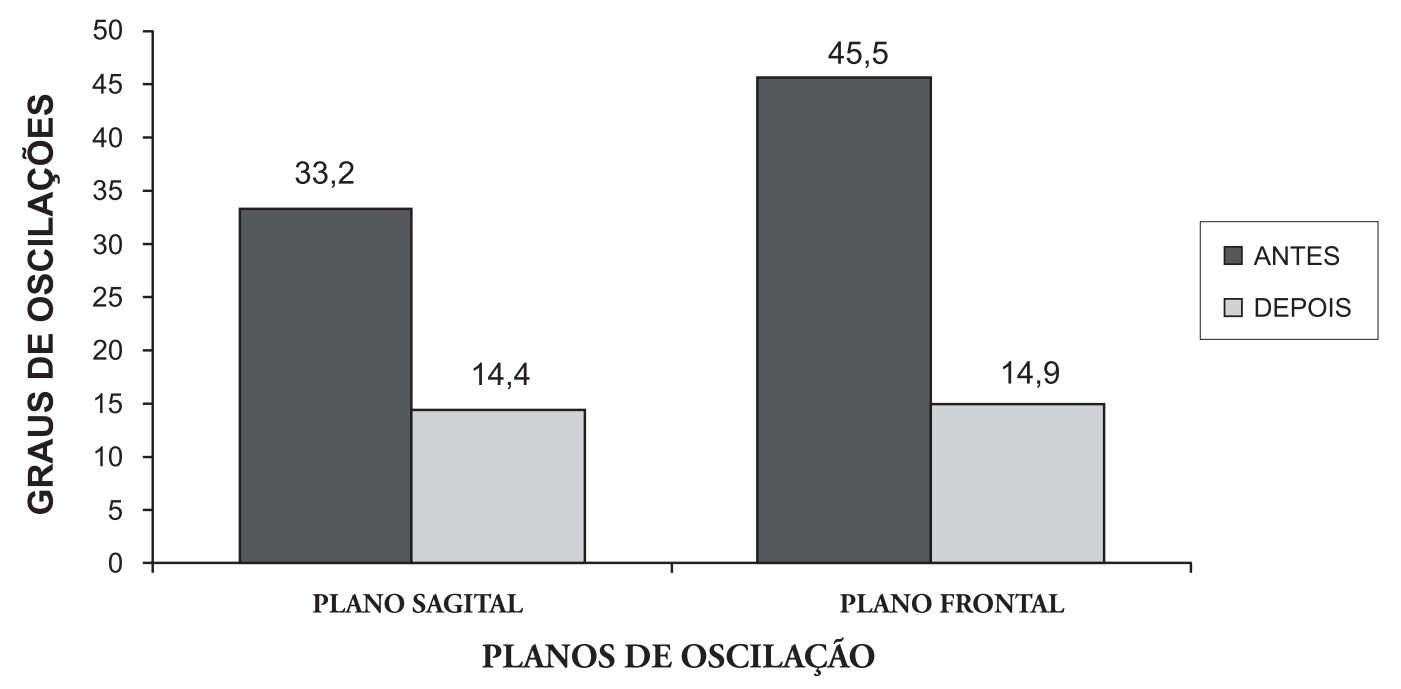

Gráfico 1. Graus de oscilação em relação aos planos Sagital e Frontal antes e após da intervenção da equoterapia.

ção dos graus de oscilações, tanto no plano frontal como no plano sagital.

Neste estudo o instrumento utilizado foi a biofotogrametria computadorizada que apresenta duas grandes vantagens na efetividade de sua aplicação clínica; baixo custo do sistema de filmagem foto para interpretação de imagens e a precisão e reprodutibilidade dos resul$\operatorname{tados}^{15-17}$. Na avaliação do equilíbrio se mostrou de fácil aplicabilidade e fidedignidade como evidenciado em vários estudos como de Meneghetti (2009) na avaliação de equilíbrio com crianças com Síndrome de Down, de Baraúna et al. (2006) em amputados, Baraúna (2003) $\operatorname{idosos}^{18-22}$.

Segundo os autores Freitas, Osvaldo e Freitas (2005); Silva et al. (2007); Freitas et al. (2005); Campos et al. (2007) os indivíduos com CMT por apresentarem deformidades nas mãos e pés, alteraçóes da sensibilidade e atrofias musculares, empenham-se em buscar o controle postural, uma vez que possuem uma importante perda sensorial e motora ${ }^{4-7}$.

Com as inúmeras alterações que ocorrem durante a prática da Hipoterapia provocadas pelos movimentos do cavalo, pelo contato com o animal, associada a uma postura nova, pode estimular a potencialidade plástica do Sistema Nervoso Central por meio de estímulos sensitivos e motores, promovendo ao praticante o mesmo mecanismo perceptivo-cognitivo-motor tornando-se assim, um recurso de grande importância ${ }^{10-14}$.

Com isso, o favorecimento de um maior controle motor, aumento do tônus muscular, a repetição do movimento, reações de equilíbrio e a percepção espaço-temporal dos vários segmentos corporais no espaço, explicaria as alteraçôes observadas ${ }^{23-25}$. Todavia, cabe ressaltar que o efeito da prática da Equoterapia é multifatorial, o que implica um conjunto de combinaçóes e ajustes por meio da qual, essa atividade exige a participaçáo de todo o corpo, contribuindo assim para o desenvolvimento da força, tônus muscular, consciência corporal, coordenação motora e para a melhorada do equilíbrio ${ }^{26-28}$.

Contudo, neste estudo, os fatores de ajustes tônicos proporcionados pela hipoterapia podem ter influenciado na melhora do controle postural, ocasionando a diminuição dos graus de oscilaçóes nos planos frontal e sagital.

É importante ressaltar como limitaçôes neste estudo que os resultados encontrados se limitam a esse caso clínico estudado.

\section{CONCLUSÃO}

Conclui-se que o indivíduo com a doença de Charcot-Marie-Tooth, apresentou melhora nas oscilaçóes do equilíbrio estático após a prática da Hipoterapia. A influência da hipoterapia, como atividade terapêutica, contribuiu para maior alinhamento biomecânico e consequentemente ativação e sinergia muscular adequada. 


\section{REFERÊNCIAS}

1.Ekman LL. Neurociência: Fundamentos para a Reabilitação. Rio de Janeiro: Koogan; 2000, 477p.

2.Teixeira E, Sauron FN, Santos LSB, Oliveira MC. Terapia Ocupacional: na Reabilitação Física. São Paulo: Roca, 2003, 571p.

3.Pires CA, Borghetti VHS, Zaffari A, Giacomini FL, Grutzmacher EL, Ferrabone JLG. Doença de Charcot-Marie-Tooth. Rev méd Hosp 1998;10(22):79-81. 4.Davies PM. Passos a Seguir: Um manual para o Tratamento da Hemiplegia no Adulto. São Paulo: Manole; 1996, 314p.

5.Freitas MRG, Osvaldo JMN, Freitas GR. Doença de Charcot-Marie-Tooth. Rev Bras Neurol 1995;31:11-21.

6.Silva DL, Neto FXP, Nunes CTA, Matos LTMB, Pacheco A. Aspectos Clínicos Otorrinolaringológicos da Doença de Charcot-Marie-Tooth. Arq. Int Otorrinolaringol 2007;11:442-76.

7.Freitas MRG, Osvaldo JMN, Maria TN, Tânia ME. Doença de CharcotMarie-Tooth: estudos eletromiográficos em 45 pacientes. Arq Neuropsiquiatr 1995;53:552-9.

\section{http://dx.doi.org/10.1590/S0004-282X1995000400002}

8.Campos AC, Santos NSA, Pereira ETK, Rocha ACF. Impacto de programa fisioterapêutico no desempenho funcional da criança com doença de CharcotMarie-Tooth tipo 2: estudo de caso. Fisioter Pesqui 2007;14:77-83.

9.Horak FB, Macpherson JM. Postural Orientation and equilibrium. In: Rowell, L. B.; Sherpherd, J. T. Ed. Handbook of physiology: New York: Oxford University Press; 1996, p.255-92.

10.Shumway-Cook A, Woollacott MH. Controle Postural Anormal. In: Shumway-Cook A, Woollacott MH. Controle Motor: Teoria e aplicações práticas.

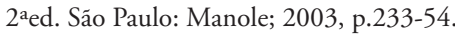

11.Medeiros M, Dias E. Equoterapia: Bases e Fundamentos. Rio de Janeiro: ed. Revinter; 2002, 51p.

12.Coimbra SAL, Bonifácio TD, Sanches, KC, Castro MFS, Jorge DA. A influência da equoterapia no equilíbrio estático e dinâmico: apresentação de caso clínico de encefalopatia não progressiva crônica do tipo diparético espástico. Fisioter Bras 2006;7:391-3.

13. Valdiviesso V, Cardillo L, Guimarães EL. A influência da equoterapia no desempenho motor e alinhamento postural da criança com paralisia cerebral espástico - atetóide. Rev Uniara 2005;16:235-41.

14.Cherng R, Liao H, Leung HWC, Hwang A. The effectiveness of therapeutic horseback riding in children with spastic cerebral palsy. Adapt Phys Activ Q 2004;21(2):103-21.

15.Krapivkin A, Nedashkovsky O, Khavkin A, Terent'eva I, Kolesnik L. Effect of intensive course of hipotherapy at children with cerebral palsy. Brain Dev 2001;23:189.

16.Ricieri DV. Biofotogrametria - Análise Cinemática Angular dos Movimentos. 2a ed.: Curitiba: Revisada e Ampliada; 2005, 89p.

17.Sanchez HM, Barreto RR, Baraúna MA, Canto RST, Morais EG. Avaliação postural de indivíduos portadores de deficiência visual através da biofotogrametria computadorizada. Fisioter Mov 2008;21:11-20.

18.Luz CCT, Souza MCO, Baraúna KMP, Guimarães MF. Avaliação postural de atletas praticantes de musculação através da biofotogrametria computadorizada: um estudo de caso. Rev FisioBrasil 2008;11:23-30.

19.Meneghetti CHZ, Blascovi-Assis SM, Deloroso FT, Rodrigues GM. Avaliação do equilíbrio estático de crianças e adolescentes com síndrome de Down. Rev Bras Fisioter 2009;13:230-5. http://dx.doi.org/10.1590/S1413-35552009005000029

20.Baraúna MA, Duarte F, Sanchez HM, Canto RST, Maluá S, Silva CDC, et al. Avaliação do Equilíbrio Estático em Indivíduos Amputados de Membros Inferiores através da Biofotogrametria Computadorizada. Rev Bras Fisioter 2006;10:83-90.

21.Baraúna MA, Canto RST, Oliveira AS, Soares AB, Silva CDC, Cardoso FAG. Avaliação do equilíbrio estático do portador de diabetes mellitus pela biofotogrametria. Diabetes Clínica 2003;7(1):57-62.

22.Baraúna MA, Barbosa SEM, Canto ST, Silva RAV, Silva CDC, Baraúna KMP. Estudo do equilíbrio estático de idosos e sua correlação com quedas. Fisioter Bras 2004;5:136-41.

23.Ernesto TT, Júnior CPL, Ávilac SN. O uso da equoterapia como recurso terapêutico para melhora do equilíbrio estático em indivíduos da terceira idade. Rev Bras Geriatr Gerontol 2008;11:391-403.

24.Santos SLM. Fisioterapia na Equoterapia: Análise de seus efeitos sobre o portador de necessidades especiais. 2aed., Aparecida: Idéias \& Letras; 2005, 127p. 25.Sartoreto EM, Silveira MM, Wibelinger LM. Equoterapia como recurso terapêutico no equilíbrio de deficientes visuais. Rev FisioBrasil 2009;12:20-4. 26.Mangili GG, Thomas PI, Silva C, Poletti S. Influência da equoterapia na função motora na paralisia cerebral diparética. Rev Fisioter Ser 2006;1:138-42. 27.Benda W, McGibbon NH, Grant KL. Improvements in muscle symmetry in children with cerebral palsy after equine-assisted therapy (hippotherapy). J Altern Complement Med 2003;9(6):817-25. http://dx.doi.org/10.1089/107555303771952163

28.Copetti F, Mota CB, Graup S, Menezes KM, Venturini EB. Comportamento angular do andar de crianças com síndrome de Down após intervenção com equoterapia. Rev Bras Fisioter 2007;11(6):503-7. 